Review Article

\title{
Gut Microbiota in Alzheimer's Disease, Depression, and Type 2 Diabetes Mellitus: The Role of Oxidative Stress
}

\author{
Maria Luca $\mathbb{D}^{1},{ }^{1}$ Maurizio Di Mauro $\mathbb{D}^{2},{ }^{2}$ Marco Di Mauro $\mathbb{D}^{3},{ }^{3}$ and Antonina Luca $\mathbb{D}^{1}$ \\ ${ }^{1}$ Department of Medical, Surgical Sciences and Advanced Technologies "GF Ingrassia", University of Catania, Italy \\ ${ }^{2}$ Department of Clinical and Experimental Medicine, University of Catania, Italy \\ ${ }^{3}$ Department of Biomedical and Biotechnological Sciences, University of Catania, Italy \\ Correspondence should be addressed to Antonina Luca; antolucaster@gmail.com
}

Received 11 November 2018; Accepted 13 March 2019; Published 17 April 2019

Academic Editor: Victor M. Victor

Copyright (c) 2019 Maria Luca et al. This is an open access article distributed under the Creative Commons Attribution License, which permits unrestricted use, distribution, and reproduction in any medium, provided the original work is properly cited.

Gut microbiota consists of over 100 trillion microorganisms including at least 1000 different species of bacteria and is crucially involved in physiological and pathophysiological processes occurring in the host. An imbalanced gastrointestinal ecosystem (dysbiosis) seems to be a contributor to the development and maintenance of several diseases, such as Alzheimer's disease, depression, and type 2 diabetes mellitus. Interestingly, the three disorders are frequently associated as demonstrated by the high comorbidity rates. In this review, we introduce gut microbiota and its role in both normal and pathological processes; then, we discuss the importance of the gut-brain axis as well as the role of oxidative stress and inflammation as mediators of the pathological processes in which dysbiosis is involved. Specific sections pertain the role of the altered gut microbiota in the pathogenesis of Alzheimer's disease, depression, and type 2 diabetes mellitus. The therapeutic implications of microbiota manipulation are briefly discussed. Finally, a conclusion comments on the possible role of dysbiosis as a common pathogenetic contributor (via oxidative stress and inflammation) shared by the three disorders.

\section{Introduction}

1.1. Gut Microbiota. Recent technological advances have increased the interest on the relationship between the microorganisms inhabiting the gut (gut microbiota) and human health. The gastrointestinal tract hosts over 100 trillion microorganisms including at least 1000 different species of bacteria [1]. In humans, about $1 / 3$ of gut microbiota is "common," while the other $2 / 3$ is different from one individual to another, providing our "personal identity" [2]. Despite the difficulties in defining a "good" microbiota, data suggest that, in adulthood, a healthy microbiota is characterized by the community stability and the species diversity. More specifically, despite lifestyle and food changes, the Firmicutes (such as Lactobacillus) and the Bacteroides represent the main bacterial phyla in the gut [3] followed by Proteobacteria, Actinobacteria (such as Bifidobacterium), and Cyanobacteria [2], which constitute an ecological community entertaining a beneficial relationship with the host [4]. As a result, an imbalance of the intestinal bacteria representation (dysbiosis) could lead to different diseases, ranging from inflammatory bowel disease to obesity, diabetes, and asthma, as well as Parkinson's disease, Alzheimer's disease (AD), and depression [5].

1.2. The Gut-Brain Axis. The term "gut-brain axis" refers to a crosstalk between the brain and the gut involving multiple overlapping pathways, including the autonomic, neuroendocrine, and immune systems as well as bacterial metabolites and neuromodulatory molecules [6].

In particular, millions of nerves end in the gastrointestinal tract mucosa, constituting the enteric nervous system which regulates the intestinal functions and communicates with the brain through the vagus nerve. The latter is responsible for the transmission of signals from the brain to the gastrointestinal tract (through the autonomic nervous system) and vice versa [7]. The presence of dysbiosis, causing the breakdown of the intestinal permeability, can lead to an inflammatory condition not limited to the gut, since the proinflammatory cytokines can get into the bloodstream 
and reach the brain [8]. The importance of inflammation should not be underestimated, since several evidences support its crucial role in several chronic disorders, such as type 2 diabetes [9], $\mathrm{AD}[10]$, and depression [11]. Apart from the cytokines, other mediators can send signals from the gut to the brain through the vagus nerve. In fact, especially after a meal rich of fats and carbohydrates, a subgroup of specialized intestinal cells, named enteroendocrine cells, releases hormones and peptides, such as 5-hydroxitryptamine (5-HT), cholecystokinin (CKK), glucagon-like peptide-1 (GLP-1), and peptide YY (PYY) [12]. These mediators exert many important functions. For example, PYY and GLP-1 inhibit the intestinal peristalsis and improve glucose metabolism attenuating pancreatic islet hypertrophy and the insulin resistance [13]. Moreover, these peptides, binding their cognate receptors located in the nucleus of the solitary tract and in the hypothalamus, induce the sense of satiety and modulate the energy expenditure [14]. In addition, GLP-1 seems to be able to upgrade hippocampal neural plasticity improving cognition [15] and to stimulate receptors located in the amygdala and in the hippocampus, thus exerting anxiolytic and antidepressant effects [16].

Interestingly, mice assuming a high-fat diet are more likely to present lower levels of short-chain fatty acids (SCFAs) than rodents assuming a low-fat diet [17]. Moreover, low levels of butyrate have been related with a higher risk to develop type 2 diabetes in humans [18]. SCFAs are involved in neurotransmission, since they modulate the synthesis of several neurotransmitters regulating behaviour and cognition. In particular, both butyric and propionic acid enhance the expression of tyrosine and tryptophan hydroxylase, enzymes involved in the synthesis of dopamine, noradrenaline, and serotonin [19]. Interestingly, evidence suggests that several bacteria could directly produce neurotransmitters, including gamma-aminobutyric acid (GABA) and serotonin. Animal studies demonstrate that Lactobacillus rhamnosus is able to modulate GABA receptor expression in the brain, thus exerting a beneficial role in the treatment of mood disorders [20]. Concerning serotonin, it is mainly produced by the gut enterochromaffin cells and modulates several physiological processes (i.e., mood regulation, sleep, and sexual behaviour). Literature data suggest that Escherichia and Enterococcus are able to produce serotonin both directly and through the production of SCFAs [21].

In summary, gut microbiota and brain are strictly intertwined and communicate through different ways, including the production of bacteria metabolites, cytokines, and neurotransmitters $[4,19,20]$. On this ground, it is not surprising that it has been hypothesized that the gut microbiota could play a pivotal role in the pathogenesis of chronic disorders such as depression, $\mathrm{AD}$, and diabetes.

1.3. Microbiota, Inflammation, and Oxidative Stress. Among the various functions of gut microbiota, the regulation of oxidative stress (OS) is probably the most fascinating one [22]. The gastrointestinal tract is rich of sources of nitric oxide (NO), such as intestinal tissues (i.e., mast cells, smooth muscle, and neural plexus), leukocytes, and commensal anaerobes. It has been demonstrated that a high nitrate intake increases both nitrite concentration and NO. In fact, gut lactobacilli and bifidobacteria are able to convert nitrate and nitrite in NO while enhancing the release of NO by host epithelial cells [23]. In addition to NO production from nitrate, gut streptomycetes and bacilli produce NO through their NO synthetase (NOS) from L-arginine [23]. NO is the principal neurotransmitter of the nonadrenergic, noncholinergic enteric nervous system and is released by the activation of NMDA receptors by glutamate. While nanomolar concentrations of NO exert a neuroprotective function being involved in signaling and apoptosis, excessive NO production is noxious, being associated with neuroinflammation, cellular damage, axonal degeneration, and neurodegenerative disorders [10]. An aberrant production of NO leads to detrimental effects due to the generation of reactive oxygen species (ROS), such as superoxide anions (which form the highly reactive peroxynitrite ion, responsible for protein nitrotyrosilation and inhibition of mitochondrial functions) and hydrogen peroxide, which forms the highly reactive hydroxyl radical that is responsible for lipid peroxidation and DNA damage [24]. The main site of the production of ROS and reactive oxygen nitrogen species (RONS) is represented by the mitochondria, primary energy centre involved in the oxidative reactions leading to adenosine triphosphate (ATP) generation. Recent studies have underlined the existence of an intertalk between host and microbiota mediated by mitochondria $[25,26]$. Gut microbiota metabolites, SCFAs in particular, influence mitochondrial function reducing ROS production [27]. Other studies have reported that SCFAs are also associated with a reduction of telomere shortening and DNA damage by altering chromatin structures and inducing the production of the antioxidant glutathione [28] and increasing COX-2 activity [29].

If on the one hand, commensal bacteria exert beneficial effects against OS, on the other hand, pathogens (i.e., Salmonella and E. coli) are able to degrade sulphur amino acids leading to hydrogen sulfide $\left(\mathrm{H}_{2} \mathrm{~S}\right)$ production in the gut. High levels of $\mathrm{H}_{2} \mathrm{~S}$ are responsible of several negative effects in the host, such as the inhibition of COX activity and the shifting of the metabolism towards glycolysis, thus leading to an increased lactate and decreased ATP production [30]. Moreover, it has been demonstrated that the exposure to high levels of $\mathrm{H}_{2} \mathrm{~S}$ leads to a decreased mitochondrial oxygen consumption and an overexpression of proinflammatory mediator genes, IL-6 in particular [31].

Considering the important role of the functionally microbiota-derived active substance on host immunological and inflammatory functions, it is apparent that the maintenance of a "healthy microbiota" becomes fundamental for the individual's wellbeing. See Figure 1 for a visual presentation of how dysbiosis favors OS.

\section{Alzheimer's Disease}

Epidemiological studies reported that 50 million people are affected by dementia worldwide [32] with an age-specific incidence ranging from 5 per 1000 at the age 65-70 to 80 per 1000 for individuals older than 85 years [33]. 


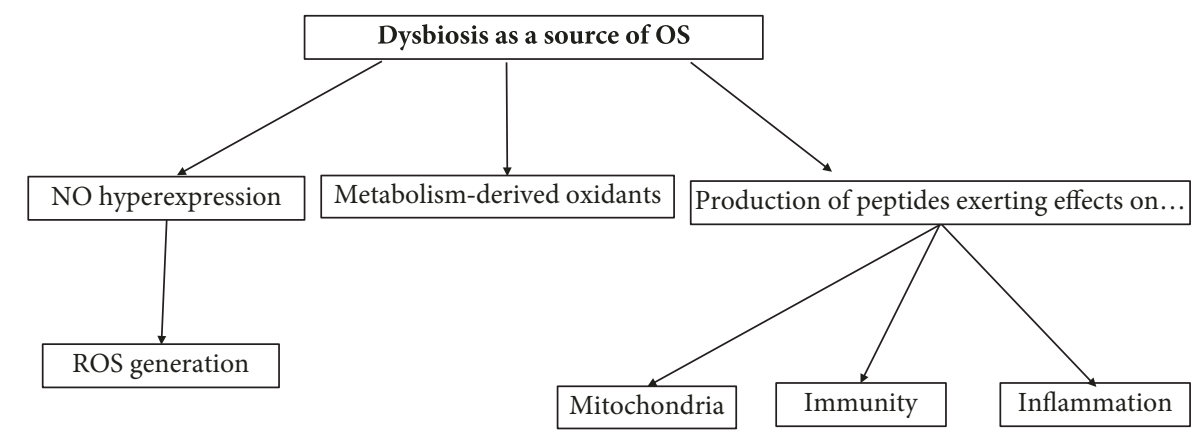

FIGURE 1: Dysbiosis favors oxidative stress and affects the immunological and inflammatory status of the host. NO: nitric oxide; OS: oxidative stress; ROS: reactive oxygen species.

$\mathrm{AD}$ is the most frequent neurodegenerative disorders and form of dementia in the elderly [34].

During the early stages of the "typical" form of $\mathrm{AD}$, the main symptom is represented by episodic memory impairment then accompanied by other cognitive domain deficits (visuospatial, attention, language, and executive functions) leading to a loss of abilities of daily living and dementia [35].

To date, thanks to biomarker research, $\mathrm{AD}$ is considered a slowly chronic progressive brain disease that can be diagnosed several years before the clinic onset, during a "preclinical stage" [36]. In fact, the pathological hallmarks of AD, including hippocampus atrophy, extracellular amyloid- $\beta$ $(\mathrm{A} \beta)$ plaques, and intracellular neurofibrillary tangles of hyperphosphorylated tau protein can be identified up to decades before the occurrence of cognitive decline and behavioural disturbances [37].

Despite some conflicting results, the role of several genetic [38, 39] and modifiable environmental risk factors in the pathogenesis of $\mathrm{AD}$ has been documented [40]. Among the modifiable risk factors, special attention has been given to the role of gut microbiome alteration in the maintenance of the chronic age-related low inflammation [41].

\subsection{Oxidative Stress and Alzheimer's Disease: The Role of Gut} Microbiome. From a pathophysiological point of view, as mentioned before, the $\mathrm{AD}$ brain is characterized by $\mathrm{A} \beta 1-42$ aggregation and neurofibrillary tangles with a consequent immune response activation driven by activated microglia. This immune response activation initially allows $\mathrm{A} \beta$ clearance, but, during aging, it undergoes alterations thus leading to a progressive deposition of $A \beta$ plaques which inexorably determines synaptic dysfunction, neuron death, neuroinflammation, and OS [42].

In particular, in the $\mathrm{AD}$ brain, several sources of ROS production have been demonstrated: (1) mitochondrial dysfunction determined by a cytochrome $\mathrm{c}$ oxidase deficiency [43] as well as by an alteration of their permeability due to the OS-related hyperactivation of glycogen synthase kinase (GSK-3) [44], (2) endoplasmic reticulum dysfunction due to its engagement in the elimination of abundant misfolded proteins, such as hyperphosphorylated tau protein [45], (3) metal ions (i.e., copper, iron, and zinc) accumulated in the neuritic plaques [46], and (4) age-related microglial cell hyperactivation with a subsequent high expression and activation of NADPH oxidase resulting in overproduction of hydrogen peroxide [47].

It should be also emphasized that the CNS is particularly vulnerable to OS for several reasons, including its high oxygen consumption, its use of different reactive species in the signalling process, and its scarce antioxidant metabolism [48].

Although it is still debated whether OS represents the determinant or the immediate consequence of the neurodegenerative processes, OS is indubitably involved in the key events driving the progressive neuronal loss [10]. Markers of lipid peroxidation have been, in fact, detected in biological samples of both $\mathrm{AD}$ animal models [49] and patients [50] as well as high levels of protein oxidation markers, such as carbonyls [51]. In this scenario, while a "well-balanced" gut microbiota seems to exert a positive role in the reduction of ROS production via SCFA such as N-butyrate [27], a dysbiosis may lead to systemic inflammation determining, over the years, microglia activation, BBB damage, and consequent crossing of pathogens and immune cells [52].

The most recent theories, in fact, consider AD not only as a result of a confined brain inflammation but also as the consequence of peripheral inflammatory reaction [53]. To support these theories, an association between antimicrobial response and $\mathrm{A} \beta$ production has been reported. Indeed, $\mathrm{A} \beta$ exerts an antimicrobial peptide role against several pathogens such as bacteria [54] and viruses [55]. It could therefore be hypothesized a periodic, growing with aging, $\mathrm{A} \beta$ production following new infections or reactivation of latent brain infections. Moreover, it has been demonstrated that some microbes are able to contribute to $\mathrm{A} \beta$ accumulation producing, themselves, a microbial amyloid. The latter, being similar to the cerebral one, easily reaches the brain due to the age-related increased permeability of gastrointestinal mucosa and BBB; being recognized by the immune cells, microbial amyloid determines a massive release of inflammatory cytokines thus sustaining chronic inflammation in $\mathrm{AD}[41]$.

Unfortunately, it has been demonstrated that the agerelated reduction of microbiota biodiversity, with the relative abundance of Proteobacteria and decrease of Bifidobacteria, contributes to the occurrence of dementia not only through the significant reduction of beneficial SCFAs but also through interfering with lipid metabolism. In 
particular, Bifidobacteria could exert a fundamental hypocholesterolemic role both directly, reducing the absorption and production of cholesterol and facilitating its faecal elimination [56], and indirectly, increasing the serum levels of leptin, an antiobesity hormone recently associated with hippocampal long-term potentiation and memory impairment prevention [57, 58]. Considering that lipids exert a fundamental role in APP trafficking and processing thus influencing the $\mathrm{A} \beta$ oligomer production, the maintenance of microbiota biodiversity appears to be fundamental. Moreover, it should be noted that a dysfunctional lipid metabolism has been found to be associated with anxiety [59], which has been in turn linked to subcortical amyloidosis in nondemented patients [60].

A recent double-blind controlled trial demonstrated that treating $\mathrm{AD}$ patients with a probiotic formulation containing Lactobacilli and Bifidobacteria normalized the serum triglyceride levels and improved cognitive performances, thus confirming the role of microbiota in the maintenance of a balanced lipid metabolism [61].

Another mechanism through which microbiota protects against cognitive impairment is represented by the documented ability of Lactobacilli to reduce ammonia concentration in rats [62].

Ammonia is one of the end-products of protein catabolism, historically implicated in the pathogenesis of $\mathrm{AD}$ [63]. Due to its neurotoxic nature even at low concentrations, an efficient astrocyte-modulated brain ammonia detoxification through the formation of glutamine by glutamine synthetase (GS) is crucial [64]. With aging, a lower brain GS activity has been demonstrated with the consequent elevation of ammonia concentration. In turn, hyperammonemia causes a progressive mitochondrial dysfunction, thus determining an increase in ROS production and superoxidase levels, while favoring the decrease of cytochrome $c$ oxidase, superoxide dismutase, and glutathione peroxidase [65].

In conclusion, in an era marked by therapeutic failures despite scientific efforts, deepening the knowledge of the diversified mechanisms through which microbiota could exert a role in counteracting cognitive impairment appears to be of fundamental importance. Further research on the effectiveness of microbiota manipulation as a therapeutic tool is needed.

\section{Depression}

Depression is probably the most common mental disorder, affecting more than $17 \%$ of the American general population [66]. It is characterized by depressed mood, apathy, anhedonia, sleep disturbances, appetite or weight changes, psychomotor retardation [67] or agitation, cognitive impairment, thoughts of guilt, and recurrent thoughts about death or suicide [68]. This mental disorder, strongly associated with fatigue, loss of productivity, and increased mortality, represents an economic burden for public health [69].

Even if the aetiology of depression is still unclear, several neurobiological mechanisms seem to play a role in its occurrence such as (1) the reduction of serotonin, norepinephrine, and dopamine; (2) the alteration of the hypothalamic- pituitary-adrenal (HPA) axis with the consequently elevated plasmatic cortisol level; and (3) the imbalance between proinflammatory and anti-inflammatory mediators [70]. As discussed below, both animal and human studies have demonstrated a certain role of an "altered" microbiota in this biological scenario.

3.1. Gut Microbiota in Depression: The Role of Oxidative Stress. Several studies have reported an imbalance between elevated levels of proinflammatory cytokines, such as IL-1, IL-6, IL-8, IL-12, tumor necrosis factor-alpha (TNF- $\alpha$ ), and decreased level of anti-inflammatory cytokines, such as transforming growth factor-beta and IL-10 in patients suffering from depression [70, 71]. Moreover, nonspecific inflammatory markers (i.e., acute phase protein, $\alpha 1$-antitrypsine, haptoglobin, fibrinogen, and C-reactive protein) have been found to be high in depressed patients [72].

From a biochemical point of view, as mentioned before, the activation of the inflammatory pathway is characterized by a hyperproduction of reactive oxygen species (ROS) and reactive nitrogen species (RNS) with a consequent damage of DNA, proteins, mitochondria, and cell membranes [73]. The presence of an oxidative and nitrosative stress is supported by the detection in depressed patients of high levels of by-products of lipid peroxidation such as malondialdehyde and 4-hydroxynonenal [74]. Moreover, if on the one hand, depression is characterized by an increased oxidative and nitrosative stress pathway, on the other hand, endogenous antioxidants such as zinc, glutathione, coenzyme Q10, melatonin, and vitamin $\mathrm{E}$ have been found to be decreased. These aforementioned substances, involved in the mitochondrial functioning and in the regulation of cAMP/circadian gene, may lead, if deficient, to neurodegeneration and decreased neurogenesis and neuroplasticity [75]. Probably, the major expression of an "altered" gut microbiota lays just in these oxidative mechanisms. In fact, while germ-free mice present a reduced antioxidant enzyme activity (i.e., catalase, glutathione peroxidase, and superoxide) [76, 77], an altered microbiota can stimulate the NADPH oxidase [78] and the NO synthesis [79], thus inducing OS.

The "depression-associated bacteria" are able to induce depressed mood both directly, producing valeric acid (adenosine A1 receptor inverse agonist) [80], and indirectly, promoting the production of kynurenine from tryptophan [81].

Moreover, while in physiological conditions, gut microbiota is separated from the systemic immune system by the epithelial barrier, in pathological conditions, it can reach the mesenteric lymph nodes determining the activation of monocytes and macrophages and the consequent production of inflammatory mediators with antibacterial properties, such as lysozyme [82].

In normal conditions, the gut epithelial barrier is protected from OS and inflammation by a class of ubiquitously expressed intracellular proteins, the heat shock proteins (HSP). These proteins are chaperones and play a role in the synthesis and folding of other proteins [83].

Due to their role in the repair and stabilization of proteins, evidence suggests that the synthesis of the HSPs is strongly enhanced in physical and psychological stressful 
conditions [84]. Nevertheless, if on the one hand, the upregulation of the HSPs can be considered as a cellular "defence" mechanism, on the other hand, their release in the extracellular matrix, occurring during cellular necrosis or apoptosis, can be remarkably harmful. Extracellular HSPs, in fact, are able to stimulate an inflammatory response leading to an increased proinflammatory cytokine secretion [84]. The demonstration of high plasmatic concentration of extracellular HSP70 in depressed patients has led to hypothesize that it could play a role in the occurrence of mood disorders [85]. Interestingly, the physiological epithelial HSP tone is influenced by gut microbiota activity and diversity. Several Bifidobacteria and Lactobacilli are, in fact, strong inducers of gut epithelial HSPs, thus contributing to gut protection [86].

The relationship between depression and microbiota has been known for several decades, when animal studies reported that stressed mice presented marked reductions of the number of gut lactobacilli [87]. Recently, human studies have confirmed this early observation, reporting a less represented microbial diversity, with a relative abundance of Bacteroidetes and a reduction of Lachnospiraceae, in depressed patients [88]. In addition, chronic depressed subjects present high plasmatic levels of immunoglobulin (Ig) A and IgM antibodies against the LPS of Enterobacteriaceae, thus supporting the hypothesis that microbiota could play a role in the occurrence of depression probably sustaining a chronic inflammatory status [82]. Moreover, stressed germfree mice present high circulating levels of ACHT and corticosterone, depression-sustaining hormone [89].

On these grounds, even if literature data still report conflicting results, the interest in treating depression through the administration of probiotics is growing so that the term "psychobiotics" is increasingly used. However, despite probiotics are able to decrease the plasmatic levels of cortisol with a consequent psychological wellbeing and a reduction of depressive symptoms [90], literature data are still inconsistent. In fact, while some studies demonstrated the efficacy of probiotic augmentation in the reduction of depressive symptoms in drug-resistant patients $[91,92]$, the administration of probiotics alone seems to be ineffective [93].

Nonetheless, it is undeniable that the potential usefulness of probiotics in major depression is a fascinating and worthy of investigation topic, also considering the elevated tolerability of these compounds.

\section{Type 2 Diabetes Mellitus}

Diabetes and obesity, two world epidemics, represent a global challenge for health care [94].

The 2016 Global Report on Diabetes by the World Health Organization (WHO) states that in 2014 the prevalence of diabetes reached up to $8.5 \%$ in the adult population, while more than 1 in 10 adults were obese [95]. Diabetes mellitus was the $7^{\text {th }}$ cause of death in 2016 and is projected to move up in the rankings by $2030[96,97]$.

Hence, research pertaining obesity, metabolic syndrome, and type 2 diabetes mellitus (T2DM) has been focusing on the identification of potentially modifiable dysfunctions, among which dysbiosis of the gut microbiota has been attracting much interest [98-101].

As previously stated in this review, gut microbiota plays a fundamental role in physiological and pathophysiological processes occurring in the host and it is apparent how diet could directly influence the fine balance of the intestinal ecosystem [102-105].

4.1. Gut Microbiota in Obesity, Insulin Resistance, and T2DM: The Role of Oxidative Stress. Obese individuals are frequently characterized by insulin resistance, a condition associated with low-grade subclinical inflammation leading to hyperglycemia and favoring the onset of T2DM [106].

An animal-model study demonstrated how gut microbiome transplant from twin mice discordant for obesity to germ-free mice influenced the metabolic arrangement of the host. More specifically, mice receiving faecal transplant from obese donors had a higher tendency to develop obesity compared to mouse receiving the transplant from a lean donor. Interestingly, diet modulated the colonizing of the microbiome and acted as a protector from developing the obesity phenotype [107].

Differences between obese and lean people both in terms of concentration and type of resident bacterial population have been reported. Overall, individuals with a low bacterial richness are characterized by marked adiposity, insulin resistance, and dyslipidaemia [108]. Firmicutes and Bacteroides phyla account for the $90 \%$ of the adult gut flora. An imbalance between the two species has been linked to obesity with conflicting results, but it seems that a reduced production of the microbiota-induced fermentation product butyrate (exerting anti-inflammatory and antioxidative properties and increasing insulin sensitivity in mice) and an increased release of LPS (already described as endotoxin) could favor obesity; the latter is characterized by a microbiota prone to harvest energy from diet $[109,110]$.

Concerning T2DM, gut alterations lead to an enrichment in membrane transport of sugars, branched-chain amino acid transport and sulfate reduction, decreased butyrate biosynthesis, and OS response that could account for the proinflammatory state characterizing diabetes. Interestingly, patients suffering from T2DM show a dysbiotic condition characterized by a decrease of butyrate-producing bacteria (such as Firmicutes, Roseburia intestinalis, and Faecalibacterium prausnitzii) and an increase in opportunistic pathogens. Despite this unfavorable condition, the gut environment of T2DM attempts to limit OS, with an increase in functions related to OS resistance, such as catalase, peroxiredoxin, and glutathione reductase. In general, despite the role of some metabolites (such as butyrate) needs to be further confirmed, it has been hypothesized that dysbiosis in T2DM could be related to qualitative, more than quantitative, changes in the gastrointestinal ecosystem and a condition of OS that could increase the risk of diabetes complications [101, 111].

While scientific evidence supports the link between gut microbiota and metabolic syndrome [100], the relationship between inflammation, gut microbiome, and metabolic alterations has not yet been clearly elucidated. 
A potential interconnection between these three factors could be represented by diet; indeed, it has been reported that a high-fat diet leads to an increase in the proportion of lipopolysaccharide- (LPS-) containing microbiota and in the LPS plasma levels, thus leading to the so-called endotoxemia. The latter contributes to the occurrence of insulin resistance and accounts for chronic low-grade inflammation and OS characterizing metabolic syndrome. The circulation of endotoxins is made possible through the permeability alterations and the changes in composition affecting the gut $[112,113]$. LPS directly sustain insulin resistance and inflammation via toll-like receptor (TLR) signaling. TLR4 in particular serves as a coreceptor for the monocyte differentiation antigen CD14 and mediates the LPS-induced inflammatory cascade and innate immune response $[113,114]$.

The discussed findings have practical implications. In fact, it can be inferred that the manipulation of intestinal ecosystem could interfere with the dysbiosis-related prooxidative and proinflammatory mechanisms described above. Preliminary reports support this hypothesis. In fact, the use of antibiotics in mice has been related to reduced endotoxemia, OS, and inflammation [115]. In addition, Lactobacillus, Bifidobacterium, and Enterobacter halii are bacterial candidates for the treatment of obesity, even though more studies are needed to confirm their efficacy [116]. In terms of future perspectives, the characterization of faecal metagenome could help identifying people at high risk of developing metabolic and inflammatory complications [18].

\section{Gut-Brain Axis: Therapeutic Perspectives}

As stated above in the sections dedicated to $\mathrm{AD}$, depression, and diabetes, several studies have reported the usefulness of microbiota manipulation in the treatment of these disorders $[61,91,115]$. In recent years, special attention has been given by researchers to probiotic supplementation with promising results [117-119]. Despite further insights on the efficacy of dysbiosis restoration in these disorders are needed, the gutbrain axis cannot be simply dismissed as a "fashionable topic" [120].

\section{Conclusion}

Dysbiosis has been demonstrated to exert regulatory functions on inflammation and OS and represents a pathogenetic contributor shared by AD, depression, and T2DM $[8,22,41$, $81,101]$, three disorders characterized by a prooxidative and proinflammatory condition $[42,70,111]$. The gut-brain axis can account for the molecular similarities linking these disorders, also confirmed by the high rates of comorbidity between depression and T2DM, which in turn increase the risk of dementia. Metabolism, cognition, and mood are strictly intertwined; if glucose toxicity can directly interfere with the cognitive functions, the insulin pathway is involved in amyloid formation, while depression can precipitate neuronal damage through inflammatory mechanisms [121]. Deepening the knowledge on the pathogenetic mechanisms of these burdening disorders could open new scenarios. In fact, the manipulation of the gut environment could be further investigated as a preventive and/or therapeutic tool with (potentially) a good safety profile.

\section{Conflicts of Interest}

The authors declare that there is no conflict of interests regarding the publication of this paper.

\section{Authors' Contributions}

Maria Luca and Maurizio Di Mauro contributed equally to this work.

\section{References}

[1] S. Bermon, B. Petriz, A. Kajeniene, J. Prestes, L. Castell, and O. L. Franco, "The microbiota: an exercise immunology perspective," Exercise Immunology Review, vol. 21, pp. 70-79, 2015.

[2] MetaHIT Consortium, J. Qin, R. Li et al., "A human gut microbial gene catalogue established by metagenomic sequencing," Nature, vol. 464, no. 7285, pp. 59-65, 2010.

[3] E. K. Costello, C. L. Lauber, M. Hamady, N. Fierer, J. I. Gordon, and R. Knight, "Bacterial community variation in human body habitats across space and time," Science, vol. 326, no. 5960, pp. 1694-1697, 2009.

[4] J. K. Nicholson, E. Holmes, J. Kinross et al., "Host-gut microbiota metabolic interactions," Science, vol. 336, no. 6086, pp. 1262-1267, 2012.

[5] E. Sherwin, T. G. Dinan, and J. F. Cryan, "Recent developments in understanding the role of the gut microbiota in brain health and disease," Annals of the New York Academy of Sciences, vol. 1420, no. 1, pp. 5-25, 2018.

[6] E. M. M. Quigley, "Microbiota-brain-gut axis and neurodegenerative diseases," Current Neurology and Neuroscience Reports, vol. 17, no. 12, p. 94, 2017.

[7] M. M. Moriss, "Vagus nerve," in Encyclopedia of Science, Salem Press, 2013.

[8] J. R. Kelly, P. J. Kennedy, J. F. Cryan, T. G. Dinan, G. Clarke, and N. P. Hyland, "Breaking down the barriers: the gut microbiome, intestinal permeability and stress-related psychiatric disorders," Frontiers in Cellular Neuroscience, vol. 9, p. 392, 2015.

[9] Y. Xu, H. Zhou, and Q. Zhu, "The impact of microbiota-gutbrain axis on diabetic cognition impairment," Frontiers in Aging Neuroscience, vol. 27, no. 9, p. 106, 2017.

[10] M. Luca, A. Luca, and C. Calandra, "The role of oxidative damage in the pathogenesis and progression of Alzheimer's disease and vascular dementia," Oxidative Medicine and Cellular Longevity, vol. 2015, Article ID 504678, 8 pages, 2015.

[11] J.-M. Kim, R. Stewart, J.-W. Kim et al., "Changes in proinflammatory cytokine levels and late-life depression: a two year population based longitudinal study," Psychoneuroendocrinology, vol. 90, pp. 85-91, 2018.

[12] R. Latorre, C. Sternini, R. De Giorgio, and B. Greenwood-Van Meerveld, "Enteroendocrine cells: a review of their role in brain-gut communication," Neurogastroenterology and Motility, vol. 28, no. 5, pp. 620-630, 2016.

[13] I. Hwang, Y. J. Park, Y. R. Kim et al., "Alteration of gut microbiota by vancomycin and bacitracin improves insulin 
resistance via glucagon-like peptide 1 in diet-induced obesity," The FASEB Journal, vol. 29, no. 6, pp. 2397-2411, 2015.

[14] P. V. Bauer, S. C. Hamr, and F. A. Duca, "Regulation of energy balance by a gut-brain axis and involvement of the gut microbiota," Cellular and Molecular Life Sciences, vol. 73, no. 4, pp. 737-755, 2016.

[15] L. Li, Z. F. Zhang, C. Holscher, C. Gao, Y. H. Jiang, and Y. Z. Liu, "Val ${ }^{8}$ glucagon-like peptide-1 prevents tau hyperphosphorylation, impairment of spatial learning and ultrastructural cellular damage induced by streptozotocin in rat brains," European Journal of Pharmacology, vol. 674, no. 23, pp. 280-286, 2012.

[16] R. H. Anderberg, J. E. Richard, C. Hansson, H. Nissbrandt, F. Bergquist, and K. P. Skibicka, "GLP-1 is both anxiogenic and antidepressant; divergent effects of acute and chronic GLP-1 on emotionality," Psychoneuroendocrinology, vol. 65, pp. 54-66, 2016.

[17] K. Berger, P. Falck, C. Linninge et al., "Cereal byproducts have prebiotic potential in mice fed a high-fat diet," Journal of Agricultural and Food Chemistry, vol. 62, no. 32, pp. 8169-8178, 2014.

[18] F. H. Karlsson, V. Tremaroli, I. Nookaew et al., "Gut metagenome in European women with normal, impaired and diabetic glucose control," Nature, vol. 498, no. 7452, pp. 99-103, 2013.

[19] B. B. Nankova, R. Agarwal, D. F. MacFabe, and E. F. La Gamma, "Enteric bacterial metabolites propionic and butyric acid modulate gene expression, including CREB-dependent catecholaminergic neurotransmission, in PC12 cells-possible relevance to autism spectrum disorders," PLoS One, vol. 9, no. 8, article e103740, 2014.

[20] J. A. Bravo, P. Forsythe, M. V. Chew et al., "Ingestion of Lactobacillus strain regulates emotional behavior and central GABA receptor expression in a mouse via the vagus nerve," Proceedings of the National Academy of Sciences of the United States of America, vol. 108, no. 38, pp. 16050-16055, 2011.

[21] J. F. Cryan and T. G. Dinan, "Mind-altering microorganisms: the impact of the gut microbiota on brain and behaviour," Nature Reviews. Neuroscience, vol. 13, no. 10, pp. 701-712, 2012.

[22] J. Xu, C. Xu, X. Chen et al., "Regulation of an antioxidant blend on intestinal redox status and major microbiota in early weaned piglets," Nutrition, vol. 30, no. 5, pp. 584-589, 2014.

[23] A. V. Oleskin and B. A. Shenderov, "Neuromodulatory effects and targets of the SCFAs and gasotransmitters produced by the human symbiotic microbiota," Microbial Ecology in Health and Disease, vol. 27, article 30971, 2016.

[24] J. K. Y. Tse, "Gut microbiota, nitric oxide, and microglia as prerequisites for neurodegenerative disorders," ACS Chemical Neuroscience, vol. 8, no. 7, pp. 1438-1447, 2017.

[25] Y. Saint-Georges-Chaumet and M. Edeas, "Microbiota-mitochondria inter-talk: consequence for microbiota-host interaction," Pathogens and Disease, vol. 74, no. 1, article ftv096, 2015.

[26] E. Lobet, J. J. Letesson, and T. Arnould, "Mitochondria: a target for bacteria," Biochemical Pharmacology, vol. 94, no. 3, pp. 173-185, 2015.

[27] W. Mottawea, C. K. Chiang, M. Mühlbauer et al., "Altered intestinal microbiota-host mitochondria crosstalk in new onset Crohn's disease," Nature Communications, vol. 7, no. 1, article 13419, 2016.
[28] H. M. Hamer, D. M. A. E. Jonkers, A. Bast et al., "Butyrate modulates oxidative stress in the colonic mucosa of healthy humans," Clinical Nutrition, vol. 28, no. 1, pp. 88-93, 2009.

[29] R. Valladares, D. Sankar, N. Li et al., "Lactobacillus johnsonii N6.2 mitigates the development of type 1 diabetes in BB-DP rats," PLoS One, vol. 5, no. 5, article e10507, 2010.

[30] X. Leschelle, M. Goubern, M. Andriamihaja et al., “Adaptative metabolic response of human colonic epithelial cells to the adverse effects of the luminal compound sulfide," Biochimica et Biophysica Acta (BBA) - General Subjects, vol. 1725, no. 2, pp. 201-212, 2005.

[31] M. Beaumont, M. Andriamihaja, A. Lan et al., "Detrimental effects for colonocytes of an increased exposure to luminal hydrogen sulfide: the adaptive response," Free Radical Biology \& Medicine, vol. 93, pp. 155-164, 2016.

[32] Alzheimer's Disease International, World Alzheimer Report, 2018: The State of the Art of Dementia Research: New Frontiers, Alzheimer's Disease International (ADI), London, 2018.

[33] A. L. Sosa-Ortiz, I. Acosta-Castillo, and M. J. Prince, "Epidemiology of dementias and Alzheimer's disease," Archives of Medical Research, vol. 43, no. 8, pp. 600-608, 2012.

[34] L. C. dos Santos Picanco, P. F. Ozela, M. de Fatima de Brito Brito et al., "Alzheimer's disease: a review from the pathophysiology to diagnosis, new perspectives for pharmacological treatment," Current Medicinal Chemistry, vol. 25, no. 26, pp. 3141-3159, 2018.

[35] G. M. McKhann, D. S. Knopman, H. Chertkow et al., "The diagnosis of dementia due to Alzheimer's disease: recommendations from the National Institute on Aging-Alzheimer's Association workgroups on diagnostic guidelines for Alzheimer's disease," Alzheimers Dement, vol. 7, no. 3, pp. 263-269, 2011.

[36] B. Dubois, H. Hampel, H. H. Feldman et al., "Preclinical Alzheimer's disease: definition, natural history, and diagnostic criteria," Alzheimers Dement, vol. 12, no. 3, pp. 292-323, 2016.

[37] K. Blennow, M. J. de Leon, and H. Zetterberg, “Alzheimer's disease," Lancet, vol. 368, no. 9533, pp. 387-403, 2006.

[38] E. Bruno, G. Quattrocchi, A. Nicoletti et al., "Lack of interaction between LRP1 and A2M polymorphisms for the risk of Alzheimer disease," Neuroscience Letters, vol. 482, no. 2, pp. 112-116, 2010.

[39] D. P. Veitch, M. W. Weiner, P. S. Aisen et al., "Understanding disease progression and improving Alzheimer's disease clinical trials: recent highlights from the Alzheimer's Disease Neuroimaging Initiative," Alzheimers Dement, vol. 15, no. 1, pp. 106-152, 2019.

[40] H. Xue, Q. Sun, L. Liu et al., "Risk factors of transition from mild cognitive impairment to Alzheimer's disease and death: a cohort study," Comprehensive Psychiatry, vol. 78, pp. 91-97, 2017.

[41] Y.Zhao and W. J. Lukiw, "Microbiome-generated amyloid and potential impact on amyloidogenesis in Alzheimer's disease," Journal of Nature and Science, vol. 1, no. 7, article e138, 2015.

[42] B. Penke, F. Bogár, and L. Fülöp, " $\beta$-Amyloid and the pathomechanisms of Alzheimer's disease: a comprehensive view," Molecules, vol. 22, no. 10, article E1692, 2017.

[43] R. Castellani, K. Hirai, G. Aliev et al., "Role of mitochondrial dysfunction in Alzheimer's disease," Journal of Neuroscience Research, vol. 70, no. 3, pp. 357-360, 2002. 
[44] A. Luca, C. Calandra, and M. Luca, "Gsk3 signalling and redox status in bipolar disorder: evidence from lithium efficacy," Oxidative Medicine and Cellular Longevity, vol. 2016, Article ID 3030547, 12 pages, 2016.

[45] B. Bhandary, A. Marahatta, H. R. Kim, and H. J. Chae, "An involvement of oxidative stress in endoplasmic reticulum stress and its associated diseases," International Journal of Molecular Sciences, vol. 14, no. 1, pp. 434-456, 2013.

[46] P. A. Adlard and A. I. Bush, "Metals and Alzheimer's disease: how far have we come in the clinic?," Journal of Alzheimer's Disease, vol. 62, no. 3, pp. 1369-1379, 2018.

[47] A. Luca, C. Calandra, and M. Luca, "Molecular bases of Alzheimer's disease and neurodegeneration: the role of neuroglia," Aging and Disease, vol. 9, no. 6, pp. 1134-1152, 2018.

[48] J. N. Cobley, M. L. Fiorello, and D. M. Bailey, "13 reasons why the brain is susceptible to oxidative stress," Redox Biology, vol. 15, pp. 490-503, 2018.

[49] S. M. Raefsky, R. Furman, G. Milne et al., "Deuterated polyunsaturated fatty acids reduce brain lipid peroxidation and hippocampal amyloid $\beta$-peptide levels, without discernable behavioral effects in an APP/PS1 mutant transgenic mouse model of Alzheimer's disease," Neurobiology of Aging, vol. 66, pp. 165-176, 2018.

[50] C. Peña-Bautista, C. Vigor, J. M. Galano et al., "Plasma lipid peroxidation biomarkers for early and non-invasive Alzheimer disease detection," Free Radical Biology \& Medicine, vol. 124, pp. 388-394, 2018.

[51] L. Shen, Y. Chen, A. Yang et al., "Redox proteomic profiling of specifically carbonylated proteins in the serum of triple transgenic Alzheimer's disease mice," International Journal of Molecular Sciences, vol. 17, no. 4, p. 469, 2016.

[52] M. Sochocka, B. S. Diniz, and J. Leszek, "Inflammatory response in the CNS: friend or foe?," Molecular Neurobiology, vol. 54, no. 10, pp. 8071-8089, 2017.

[53] A. le Page, G. Dupuis, E. H. Frost et al., "Role of the peripheral innate immune system in the development of Alzheimer's disease," Experimental Gerontology, vol. 107, pp. 59-66, 2018.

[54] C. S. Little, C. J. Hammond, A. MacIntyre, B. J. Balin, and D. M. Appelt, "Chlamydia pneumoniae induces Alzheimerlike amyloid plaques in brains of BALB/c mice," Neurobiology of Aging, vol. 25, no. 4, pp. 419-429, 2004.

[55] C. Martin, B. Aguila, P. Araya et al., "Inflammatory and neurodegeneration markers during asymptomatic HSV-1 reactivation," Journal of Alzheimer's Disease, vol. 39, no. 4, pp. 849-859, 2014.

[56] Y. N. Yin, Q. F. Yu, N. Fu, X. W. Liu, and F. G. Lu, "Effects of four Bifidobacteria on obesity in high-fat diet induced rats," World Journal of Gastroenterology, vol. 16, no. 27, pp. 3394-3401, 2010.

[57] M. Eslami, B. Sadeghi, and F. Goshadrou, "Chronic ghrelin administration restores hippocampal long-term potentiation and ameliorates memory impairment in rat model of Alzheimer's disease," Hippocampus, vol. 28, no. 10, pp. 724-734, 2018.

[58] H. An, S. Park, D. Lee et al., "Antiobesity and lipid-lowering effects of Bifidobacterium spp. in high fat diet-induced obese rats," Lipids in Health and Disease, vol. 10, no. 1, p. 116, 2011.

[59] E. Pistorio, M. Luca, A. Luca, V. Messina, and C. Calandra, "Autonomic nervous system and lipid metabolism: findings in anxious-depressive spectrum and eating disorders," Lipids in Health and Disease, vol. 10, no. 1, p. 192, 2011.

[60] B. J. Hanseeuw, V. Jonas, J. Jackson et al., "Association of anxiety with subcortical amyloidosis in cognitively normal older adults," Molecular Psychiatry, 2018.

[61] E. Akbari, Z. Asemi, R. Daneshvar Kakhaki et al., "Effect of probiotic supplementation on cognitive function and metabolic status in Alzheimer's disease: a randomized, double-blind and controlled trial," Frontiers in Aging Neuroscience, vol. 8, p. 256, 2016.

[62] J. Luo, T. Wang, S. Liang, X. Hu, W. Li, and F. Jin, "Ingestion of Lactobacillus strain reduces anxiety and improves cognitive function in the hyperammonemia rat," Science China. Life Sciences, vol. 57, no. 3, pp. 327-335, 2014.

[63] M. Fisman, M. Ball, and W. Blume, "Hyperammonemia and Alzheimer's disease," Journal of the American Geriatrics Society, vol. 37, no. 11, p. 1102, 1989.

[64] Y. Jin, P. Singh, H.-J. Chung, and S.-T. Hong, "Blood ammonia as a possible etiological agent for Alzheimer's disease," Nutrients, vol. 10, no. 5, article E564, 2018.

[65] E. Kosenko, I. Solomadin, L. Tikhonova, V. Reddy, G. Aliev, and Y. Kaminsky, "Pathogenesis of Alzheimer disease: role of oxidative stress, amyloid- $\beta$ peptides, systemic ammonia and erythrocyte energy metabolism," CNS \& Neurological Disorders Drug Targets, vol. 13, no. 1, pp. 112-119, 2014.

[66] E. Bromet, L. H. Andrade, I. Hwang et al., "Cross-national epidemiology of DSM-IV major depressive episode," BMC Medicine, vol. 9, no. 1, p. 90, 2011.

[67] M. Luca, A. Luca, and C. Calandra, "Psychomotor retardation and externally oriented thinking in major depression," Neuropsychiatric Disease and Treatment, vol. 9, pp. 759766, 2013.

[68] American Psychiatric Association, Diagnostic and Statistical Manual of Mental Disorders, Fifth Edition (DSM-5), American Psychiatric Association, Arlington, VA, USA, 2013.

[69] H. Ghanean, A. K. Ceniti, and S. H. Kennedy, "Fatigue in patients with major depressive disorder: prevalence, burden and pharmacological approaches to management," CNS Drugs, vol. 32, no. 1, pp. 65-74, 2018.

[70] M. Luca, A. Luca, and C. Calandra, "Accelerated aging in major depression: the role of nitro-oxidative stress," Oxidative Medicine and Cellular Longevity, vol. 2013, Article ID 230797, 6 pages, 2013.

[71] Y. Dowlati, N. Herrmann, W. Swardfager et al., "A metaanalysis of cytokines in major depression," Biological Psychiatry, vol. 67, no. 5, pp. 446-457, 2010.

[72] A. Sluzewska, J. Rybakowski, E. Bosmans et al., "Indicators of immune activation in major depression," Psychiatry Research, vol. 64, no. 3, pp. 161-167, 1996.

[73] M. Maes, M. Kubera, J. C. Leunis, M. Berk, M. Geffard, and E. Bosmans, "In depression, bacterial translocation may drive inflammatory responses, oxidative and nitrosative stress (O\&NS), and autoimmune responses directed against O\&NS-damaged neoepitopes," Acta Psychiatrica Scandinavica, vol. 127, no. 5, pp. 344-354, 2013.

[74] B. Leonard and M. Maes, "Mechanistic explanations how cell-mediated immune activation, inflammation and oxidative and nitrosative stress pathways and their sequels and concomitants play a role in the pathophysiology of unipolar 
depression," Neuroscience and Biobehavioral Reviews, vol. 36, no. 2, pp. 764-785, 2012.

[75] G. Anderson and M. Maes, "Oxidative/nitrosative stress and immuno-inflammatory pathways in depression: treatment implications," Current Pharmaceutical Design, vol. 20, no. 23, pp. 3812-3847, 2014.

[76] Y. J. Hsu, C. C. Chiu, Y. P. Li et al., "Effect of intestinal microbiota on exercise performance in mice," Journal of Strength and Conditioning Research, vol. 29, no. 2, pp. 552-558, 2015.

[77] Y. Dobashi, Y. Miyakawa, I. Yamamoto, and H. Amao, "Effects of intestinal microflora on superoxide dismutase activity in the mouse cecum," Experimental Animals, vol. 60, no. 2, pp. 133-139, 2011.

[78] W. N. Lin, C. C. Lin, H. Y. Cheng, and C. M. Yang, "Regulation of cyclooxygenase-2 and cytosolic phospholipase A2 gene expression by lipopolysaccharide through the RNAbinding protein HuR: involvement of NADPH oxidase, reactive oxygen species and mitogen-activated protein kinases," British Journal of Pharmacology, vol. 163, no. 8, pp. 16911706, 2011.

[79] E. D. Chan and D. W. H. Riches, "IFN- $\gamma+$ LPS induction of iNOS is modulated by ERK, JNK/SAPK, and $\mathrm{p} 38^{\text {mapk }}$ in a mouse macrophage cell line," American Journal of Physiology. Cell Physiology, vol. 280, no. 3, pp. C441-C450, 2001.

[80] M. P. Kaster, J. Budni, M. Gazal, M. P. Cunha, A. R. S. Santos, and A. L. S. Rodrigues, "The antidepressant-like effect of inosine in the FST is associated with both adenosine A1 and A 2A receptors," Purinergic Signal, vol. 9, no. 3, pp. 481-486, 2013.

[81] J. R. Kelly, Y. Borre, C. O' Brien et al., "Transferring the blues: depression-associated gut microbiota induces neurobehavioural changes in the rat," Journal of Psychiatric Research, vol. 82, pp. 109-118, 2016.

[82] M. Maes, M. Kubera, J. C. Leunis, and M. Berk, "Increased IgA and IgM responses against gut commensals in chronic depression: further evidence for increased bacterial translocation or leaky gut," Journal of Affective Disorders, vol. 141, no. 1, pp. 55-62, 2012.

[83] S. K. Calderwood, J. Gong, and A. Murshid, "Extracellular HSPs: the complicated roles of extracellular HSPs in immunity," Frontiers in Immunology, vol. 7, p. 159, 2016.

[84] T. J. Borges, B. J. Lang, R. L. Lopes, and C. Bonorino, "Modulation of alloimmunity by heat shock proteins," Frontiers in Immunology, vol. 7, p. 303, 2016.

[85] M. A. Pasquali, B. L. Harlow, C. N. Soares et al., "A longitudinal study of neurotrophic, oxidative, and inflammatory markers in first-onset depression in midlife women," European Archives of Psychiatry and Clinical Neuroscience, vol. 268, no. 8, pp. 771-781, 2018.

[86] M. E. Arnal and J. P. Lallès, "Gut epithelial inducible heatshock proteins and their modulation by diet and the microbiota," Nutrition Reviews, vol. 74, no. 3, pp. 181-197, 2016.

[87] G. W. Tannock and D. C. Savage, "Influences of dietary and environmental stress on microbial populations in the murine gastrointestinal tract," Infection and Immunity, vol. 9, no. 3, pp. 591-598, 1974.

[88] A. Naseribafrouei, K. Hestad, E. Avershina et al., "Correlation between the human fecal microbiota and depression," Neurogastroenterology and Motility, vol. 26, no. 8, pp. 1155-1162, 2014.
[89] N. Sudo, Y. Chida, Y. Aiba et al., "Postnatal microbial colonization programs the hypothalamic-pituitary-adrenal system for stress response in mice," The Journal of Physiology, vol. 558, no. 1, pp. 263-275, 2004.

[90] S. Davari, S. A. Talaei, H. Alaei, and M. Salami, "Probiotics treatment improves diabetes-induced impairment of synaptic activity and cognitive function: behavioral and electrophysiological proofs for microbiome-gut-brain axis," Neuroscience, vol. 240, pp. 287-296, 2013.

[91] M. Bambling, S. C. Edwards, S. Hall, and L. Vitetta, “A combination of probiotics and magnesium orotate attenuate depression in a small SSRI resistant cohort: an intestinal anti-inflammatory response is suggested," Inflammopharmacology, vol. 25, no. 2, pp. 271-274, 2017.

[92] G. Akkasheh, Z. Kashani-Poor, M. Tajabadi-Ebrahimi et al., "Clinical and metabolic response to probiotic administration in patients with major depressive disorder: a randomized, double-blind, placebo-controlled trial," Nutrition, vol. 32, no. 3, pp. 315-320, 2016.

[93] A. R. Romijn, J. J. Rucklidge, R. G. Kuijer, and C. Frampton, "A double-blind, randomized, placebo-controlled trial of Lactobacillus helveticus and Bifidobacterium longum for the symptoms of depression," The Australian and New Zealand Journal of Psychiatry, vol. 51, no. 8, pp. 810-821, 2017.

[94] S. Smyth and A. Heron, "Diabetes and obesity: the twin epidemics," Nature Medicine, vol. 12, no. 1, pp. 75-80, 2006.

[95] World Health Organization, Global Report on Diabetes, World Health Organization, Geneva, Switzerland, 2016, http://www.who.int/diabetes/publications/grd-2016/en/.

[96] World Health Organization, Global Health Estimates 2016: Deaths by Cause, Age, Sex, by Country and by Region, 2000-2016, World Health Organization, Geneva, Switzerland, 2018, http://www.who.int/healthinfo/global_burden_ disease/estimates/en/.

[97] C. D. Mathers and D. Loncar, "Projections of global mortality and burden of disease from 2002 to 2030," PLoS Medicine, vol. 3, no. 11, article e442, 2006.

[98] R. E. Ley, F. Bäckhed, P. Turnbaugh, C. A. Lozupone, R. D. Knight, and J. I. Gordon, "Obesity alters gut microbial ecology," Proceedings of the National Academy of Sciences of the United States of America, vol. 102, no. 31, pp. 11070-11075, 2005.

[99] F. Backhed, H. Ding, T. Wang et al., "The gut microbiota as an environmental factor that regulates fat storage," Proceedings of the National Academy of Sciences of the United States of America, vol. 101, no. 44, pp. 15718-15723, 2004.

[100] D. Festi, R. Schiumerini, L. H. Eusebi, G. Marasco, M. Taddia, and A. Colecchia, "Gut microbiota and metabolic syndrome," World Journal of Gastroenterology, vol. 20, no. 43, pp. 1607916094, 2014.

[101] H. Tilg and A. R. Moschen, "Microbiota and diabetes: an evolving relationship," Gut, vol. 63, no. 9, pp. 1513-1521, 2014.

[102] F. Bäckhed, R. E. Ley, J. L. Sonnenburg, D. A. Peterson, and J. I. Gordon, "Host-bacterial mutualism in the human intestine," Science, vol. 307, no. 5717, pp. 1915-1920, 2005.

[103] L. Dethlefsen, P. B. Eckburg, E. M. Bik, and D. A. Relman, "Assembly of the human intestinal microbiota," Trends in Ecology \& Evolution, vol. 21, no. 9, pp. 517-523, 2006.

[104] M. Rajilic-Stojanovic and W. M. de Vos, "The first 1000 cultured species of the human gastrointestinal microbiota," 
FEMS Microbiology Reviews, vol. 38, no. 5, pp. 996-1047, 2014.

[105] D. Graf, R. di Cagno, F. Fåk et al., "Contribution of diet to the composition of the human gut microbiota," Microbial Ecology in Health and Disease, vol. 26, 2015.

[106] C. de Luca and J. M. Olefsky, "Inflammation and insulin resistance,” FEBS Letters, vol. 582, no. 1, pp. 97-105, 2008.

[107] V. K. Ridaura, J. J. Faith, F. E. Rey et al., "Gut microbiota from twins discordant for obesity modulate metabolism in mice," Science, vol. 341, no. 6150, article 1241214, 2013.

[108] MetaHIT consortium, E. le Chatelier, T. Nielsen et al., "Richness of human gut microbiome correlates with metabolic markers," Nature, vol. 500, no. 7464, pp. 541-546, 2013.

[109] C. K. Chakraborti, "New-found link between microbiota and obesity," World Journal of Gastrointestinal Pathophysiology, vol. 6, no. 4, pp. 110-119, 2015.

[110] P. J. Turnbaugh, R. E. Ley, M. A. Mahowald, V. Magrini, E. R. Mardis, and J. I. Gordon, "An obesity-associated gut microbiome with increased capacity for energy harvest," Nature, vol. 444, no. 7122, pp. 1027-1031, 2006.

[111] J. Qin, Y. Li, Z. Cai et al., "A metagenome-wide association study of gut microbiota in type 2 diabetes," Nature, vol. 490, no. 7418, pp. 55-60, 2012.

[112] P. D. Cani, J. Amar, M. A. Iglesias et al., "Metabolic endotoxemia initiates obesity and insulin resistance," Diabetes, vol. 56, no. 7, pp. 1761-1772, 2007.

[113] I. Jialal and U. Rajamani, "Endotoxemia of metabolic syndrome: a pivotal mediator of meta-inflammation," Metabolic Syndrome and Related Disorders, vol. 12, no. 9, pp. 454-456, 2014.

[114] S. J. Creely, P. G. McTernan, C. M. Kusminski et al., "Lipopolysaccharide activates an innate immune system response in human adipose tissue in obesity and type 2 diabetes," American Journal of Physiology. Endocrinology and Metabolism, vol. 292, no. 3, pp. E740-E747, 2007.

[115] P. D. Cani, R. Bibiloni, C. Knauf et al., "Changes in gut microbiota control metabolic endotoxemia-induced inflammation in high-fat diet-induced obesity and diabetes in mice," Diabetes, vol. 57, no. 6, pp. 1470-1481, 2008.

[116] N. Kobyliak, C. Conte, G. Cammarota et al., "Probiotics in prevention and treatment of obesity: a critical view," Nutrition \& Metabolism, vol. 13, no. 1, p. 14, 2016.

[117] F. Leblhuber, K. Steiner, B. Schuetz, D. Fuchs, and J. M. Gostner, "Probiotic supplementation in patients with Alzheimer's dementia-an explorative intervention study," Current Alzheimer Research, vol. 15, no. 12, pp. 1106-1113, 2018.

[118] S. Firouzi, H. A. Majid, A. Ismail, N. A. Kamaruddin, and M. Y. Barakatun-Nisak, "Effect of multi-strain probiotics (multi-strain microbial cell preparation) on glycemic control and other diabetes-related outcomes in people with type 2 diabetes: a randomized controlled trial," European Journal of Nutrition, vol. 56, no. 4, pp. 1535-1550, 2017.

[119] M. Pirbaglou, J. Katz, R. J. de Souza, J. C. Stearns, M. Motamed, and P. Ritvo, "Probiotic supplementation can positively affect anxiety and depressive symptoms: a systematic review of randomized controlled trials," Nutrition Research, vol. 36, no. 9, pp. 889-898, 2016.
[120] M. Luca, "Gut microbiota and depression: just a fashionable topic?," Journal of Depression and Anxiety Forecas, vol. 1, no. 1, p. 1008, 2018.

[121] J. D. Rosenblat, R. B. Mansur, D. S. Cha, A. Baskaran, and R. S. McIntyre, "Depression, diabetes and dementia," in Comorbidity of Mental and Physical Disorders, N. Sartorius, R. I. G. Holt, and M. Maj, Eds., vol. 179, pp. 42-53, Karger, Basel, 2015. 


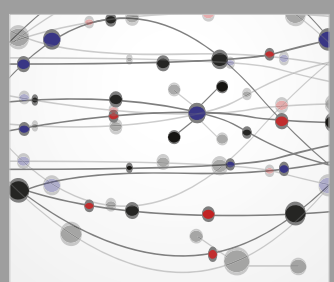

The Scientific World Journal
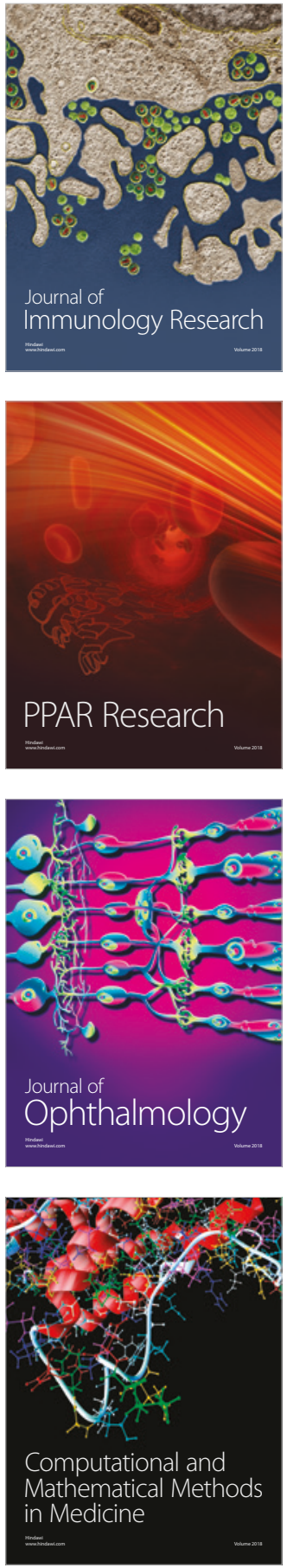

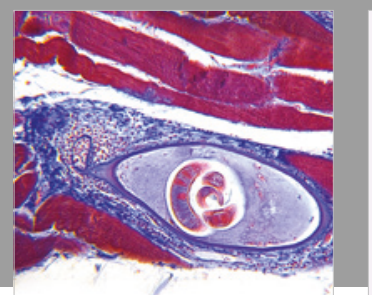

Gastroenterology Research and Practice

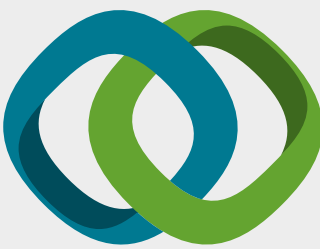

\section{Hindawi}

Submit your manuscripts at

www.hindawi.com
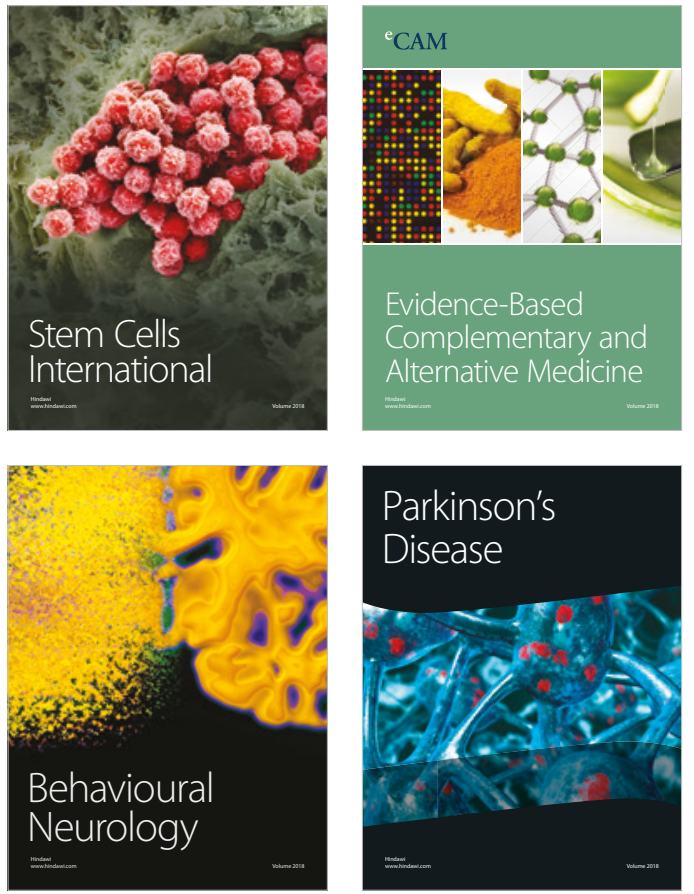

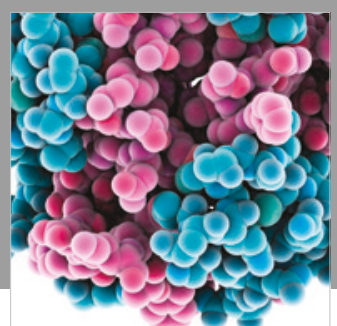

ournal of

Diabetes Research

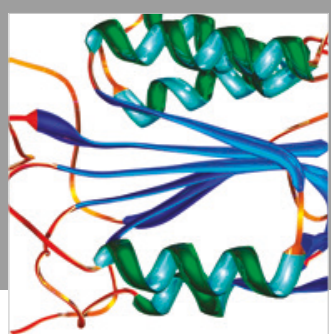

Disease Markers
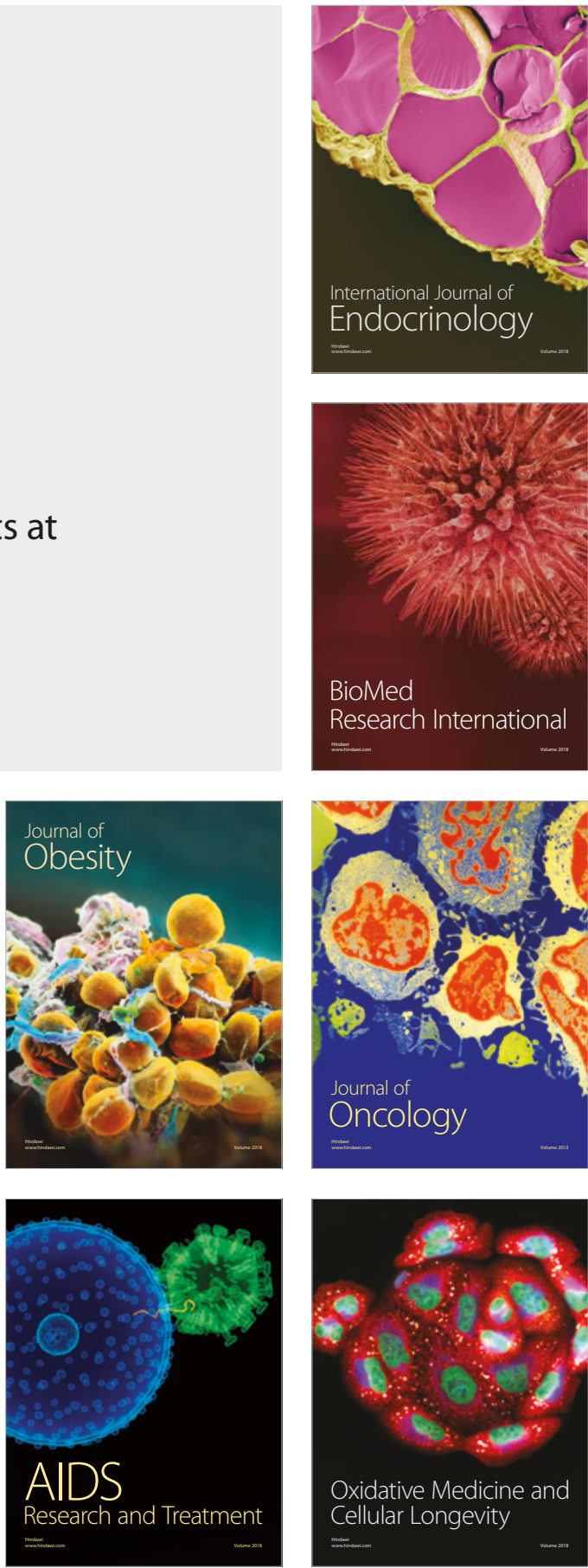